\title{
Problems in the Design of Medium and Long-term Therapeutic Trials
}

\author{
D. S. RIDLEY \\ Hospital for Tropical Diseases, London NW1 OPE
}

\begin{abstract}
The killing of Myco. leprae in vivo and its subsequent lysis are separate functions, both of which are governed by immunity. The first is also effectively achieved by chemotherapy, but it has not been shown that any of the known drugs has any effect on lysis. Any such effect is greatly outweighed by that of immunity even in lepromatous patients.

Medium and long-term drug trials in leprosy should be concerned with the general progress of the patient and the detection of relapse. It is doubtful whether there are any drugs that warrant the type of trial which is based on an assessment of bacterial lysis. Such trials are complex, time consuming and the resources available are limited. If there is any regimen that justifies such a trial present opportunities should not be wasted. In such trials the initial MI need not be a limiting factor.
\end{abstract}

I am afraid that some of the remarks I am going to make are rather obvious, even if they have not been voiced to-day already, and they will therefore be all the better for being brief. The object in bringing them up is that they raise questions which need an answer.

I refer to the problem of medium and long-term trials, that is those of more than six months duration; or rather, any trial that is designed to do more than study the effect of a drug on bacterial viability. The study of bacterial morphology combined in some cases with the more sensitive test of animal inoculation does actually test the performance of a drug in all that it can be expected to do, namely its bacteriostatic or its killing potential. This applies both to the initial effect of the drug, and also to that other acid test of its efficacy, prevention of relapse after prolonged dosage.

From the patient's point of view this leaves unresolved the important question of the lysis of bacilli, on which also hangs susceptibility to reactions. Both the killing of bacilli and their lysis are functions of immunity, though the mechanisms of the two actions are probably independent of each other. At the lepromatous end of the spectrum immunity is slight and its killing potential, if any, is greatly outweighed by drug action. But as regards lysis of bacilli it has not so far been possible to demonstrate any drug effect; and if there were any it would be heavily outweighed by the lysis due to immunity, which even in lepromatous patients is by no means negligible. Of course if one takes a drug such as rifampicin which kills bacilli perhaps two months sooner than dapsone, the rifampicin group may have the advantage over the other as regards reduction of bacilli at the end of a six 
month trial, but after a year or two the two months advantage will be imperceptible and of no account.

However, the curing of the patient is a very important matter that must somehow be assessed, and so supposing it is decided to index the fall in the number of bacilli, let us look at the "natural" background against which the effect of a therapeutic agent must be viewed. By this I mean the rate of fall on existing drug therapy, which it is assumed has no effect on bacterial numbers. The figures in the table refer to the fall in the logarithmic biopsy index, which for brevity is here called Histological Index (HI), during a period of six months treatment. It seems clear that the only group which would offer a good chance for the detection of a drug effect would be the polar LL group (LLp) in which the effect of immunity is low and it is uncomplicated by reversal reactions. In the subpolar lepromatous group (LLs or LI: it avoids confusion if this and the LLp groups are both regarded as subdivisions of LL) there are about $7.5 \%$ of reversal reactions in the first six months, about $10 \%$ in the first year and more in the second year. When a reversal reaction occurs, which may not be recognized as such clinically, the fall in the HI is increased in a variable manner to about six times on average that of a polar LL patient. The effect on the BI would be similar. With the BL group the high incidence of reversal reactions and their rather unpredictable outcome would probably make any increase in the fall in the HI due to drug action very difficult to detect. Ideally, therefore, polar LL patients should be selected for this type of trial, but as they are too few in number one uses in practice a mixture of the two forms of LL, excluding BL.

TABLE 1

Percentage fall in histological index after six months treatment in 61 patients, and percentage of patients with reversal reactions

\begin{tabular}{lccc}
\hline \% Fall HI & LLp & LLs & BL \\
\hline All cases & 5.5 & 14 & 23 \\
Cases with no reaction & 5.5 & 12 & 14 \\
Cases with reversal reaction & - & 32 & 42 \\
\% Patients with reaction & 0 & 7.5 & 33 \\
\hline
\end{tabular}

If a new drug were unexpectedly found to have a strong effect on the rate of elimination of dead bacilli, it would of course be easy to demonstrate this. A marginal effect could only be proved by using a very large number of patients, especially if the trial were only to last six months. Unfortunately large numbers are not available, and the high drop out rate would appear to exclude the possibility of long term trials at most centres. A year seems to be the most that can usefully be envisaged. Attempts to overcome the deficiency of long stay patients by undertaking multicentre trials have so far proved disappointing in their outcome. In the recent multicentre trial of low-dose clofazimine versus dapsone, organized by Ciba-Geigy, which extended over a five year period and involved at some time or other 19 centres, only 71 suitable biopsies (35 pairs) were received at six months, and fewer thereafter. This trial produces some positive results which will be published elsewhere by Dr Th. Ahrens and colleagues. But it did not encourage the belief that the multicentre approach is an easy answer to the problems posed by this sort of trial. 
My conclusions are that it is not profitable at present to attempt to measure the rate of elimination of bacilli from skin over a long period, at least at most centres, and that such a measure should not be used as a test of performance of the drugs at present available. Long-term trials should be used mainly for studies of clinical acceptability, general progress, the incidence of reactions and, in the late stage, of relapse. However, from this negative conclusion there are two positive corollaries.

(1) It is important to consider whether there are any therapeutic regimens which are worthy of a long term trial including the accurate assessment of bacterial indices. The resources throughout the world that are suitable and available for this purpose are very limited, and in view of the length of time required for such trials opportunities ought not to be wasted. Would it be useful for recommendations to be made for the guidance of any who wished to use them? Such recommendations might cover regimens considered worthy of test, if any, and possibly the protocol for a trial. In my view biopsies at stated intervals are an essential feature of such trials, for accurate classification and as the most reliable means of assessing bacterial numbers. The biopsy must extend down to the subcutis, and for the HI to be useful the granuloma in the initial biopsy must cover at least a quarter of the dermis in the section.

(2) As the bacteriostatic or bactericidal action of drugs appears to be altogether separate from the mechanism of their lysis and resorption, there is no point in stipulating a certain level of MI for trials concerned with the rate of lysis, although patients ought not to have received much treatment. It is the stipulation of a certain MI that is the cause of the exclusion of many otherwise suitable cases. The two sorts of trial ought to be regarded as separate:

\section{Immunological Agents}

The situation as regards the therapeutic testing of immunological agents such as lymphocytes and transfer factor is quite different from that of testing drugs. It would be hoped that immunological agents would not only increase the rate of bacterial lysis, but would also bring about an upgrading of immunity within the spectrum. Reactions therefore would be something to look for, instead of being a complicating factor. From this point of view the subpolar LL group would be perhaps the most useful, since patients in this group show some potential for upgrading; and at the same time the incidence of upgrading reactions on ordinary drugs is sufficiently low to make any significant increase due to an immunological agent readily detectable. 